\title{
Computational approaches of relativistic models in quantum chemistry
}

\author{
J.P. Desclaux ${ }^{\mathrm{a}}$, J. Dolbeault ${ }^{\mathrm{b}}$, M.J. Esteban $^{\mathrm{b}}$, P. Indelicato ${ }^{\mathrm{c}}$, \\ E. Séré ${ }^{b}$ \\ ${ }^{a} 15$ Chemin du Billery, F-38360, Sassenage, France. \\ E-mail: Jean-Paul.Desclaux@wanadoo.fr \\ ${ }^{\mathrm{b}}$ CEREMADE, Unité Mixte de Recherche du CNRS no. 7534 et Université Paris \\ IX-Dauphine, F-75775 Paris Cedex 16, France. \\ E-mail: dolbeaul, esteban, sere@ceremade.dauphine.fr \\ ${ }^{\mathrm{c}}$ Laboratoire Kastler-Brossel, Unité Mixte de Recherche du CNRS no. C8552, \\ École Normale Supérieure et Université Pierre et Marie Curie, Case 74, 4 place \\ Jussieu, F-75252 Paris Cedex 05, France. \\ E-mail: paul.indelicato@spectro.jussieu.fr
}

\begin{abstract}
This chapter is a review of some methods used for the computation of relativistic atomic and molecular models based on the Dirac equation. In the linear case, we briefly describe finite basis set approaches, including ones that are generated numerically, perturbation theory and effective Hamiltonians procedures, direct variational methods based on nonlinear transformations, min-max formulations and constrained minimizations. In the atomic case, we describe the MCDF method and some ways to solve numerically the homogeneous and inhomogeneous Dirac-Fock equations. Finally, we describe also some numerical methods relevant to the case of molecules.
\end{abstract}

Key words: Quantum chemistry, relativistic models for atoms and molecules, computational methods, no-pair Hamiltonian, relativistic many-body perturbation theory, relativistic random phase approximation, Dirac equation, continuous spectrum, eigenvalues, variational collapse, spurious states, finite basis, numerical basis sets, perturbation theory, effective Hamiltonians, variational methods, Rayleigh quotients, min-max, minimization, constraints, Dirac-Fock, MCDF method. 2000 MSC: 47A75, 81V45, 81V55; 65N25, 35Q40, 35Q75, 47A11, 47N50, 49R50 PACS 2001: 31.15.-p, 31.15.Ar, 31.30.Jv; 02.60.-x, 02.60.Cb, 31.15.Md, 31.15.Ne, 31.15.Pf, 31.70.-f

Preprint submitted to Elsevier Science 11 June 2002 


\section{Chapter 1. Introduction}

\section{QED and relativistic models in Quantum Chemistry}

It is now well known, following many experimental and theoretical results, that the use of $a b$ initio relativistic calculations are mandatory if one is to obtain an accurate description of heavy atoms and ions. This is true whether one is considering highly charged ions, inner shells of neutral or quasi neutral atoms or outer shells of very heavy atoms.

From a physics point of view, the natural formalism to treat such a system is Quantum Electrodynamics (QED), the prototype of field theories. For recent reviews of different aspects of QED in few electron ions see, e.g., [16,42,2]. Yet a direct calculation using only QED is impractical for atoms with more than one electron because of the complexity of the calculation. This is due to the slow rate of convergence of the so-called Ladder approximation $(1 / Z)$, that in non-relativistic theory amounts to a perturbation expansion using the electron-electron interaction as a perturbation. The only known method to do an accurate calculation is to attempt to treat to all orders the electron-electron interaction, and reserve QED for radiative corrections (interaction of the electron with its own radiation field, creation of virtual electron-positron pairs). The use of a naive approach however, taking a non-relativistic Hamiltonian and replacing one-electron Schrödinger Hamiltonian by Dirac Hamiltonian fails. This approach does not take into account one of the two main features of relativity: the possibility of particle creation, and leads to severe problems as noted already in Ref. [3] and studied in [52]. This theory, for example, does not preserve charge conservation in intermediate states and leads to divergence already in the second-order of perturbation expansion. The only way to derive a proper relativistic many-electron Hamiltonian is to start from QED. The Hamiltonian of a $\mathrm{N}$ electron system can be written formally

$$
H=H_{0}\left[N e^{-}, 0 e^{+}\right]+H_{1}\left[(N+1) e^{-}, 1 e^{+}\right]+H_{2}\left[(N+2) e^{-}, 2 e^{+}\right]+\cdots
$$

Keeping only the first term, the so-called "no-pair" Hamiltonian reads

$$
H^{\mathrm{np}}=\sum_{i=1}^{N e^{-}} h_{D}\left(r_{i}\right)+\sum_{i<j} \mathcal{U}_{i j}
$$

where (in atomic units) $h_{D}\left(r_{i}\right)=c \boldsymbol{\alpha} \cdot \boldsymbol{p}+\beta m c^{2}+V_{N}\left(\boldsymbol{r}_{i}\right)$ is a one-electron Dirac Hamiltonian in a suitable classical central potential $V_{N}$, that represents the interaction of the electron with the atomic nucleus. The speed of light is 
denoted by $c, \boldsymbol{\alpha}, \boldsymbol{\beta}$ are the Dirac matrices, with

$$
\begin{gathered}
\beta=\left(\begin{array}{cc}
\mathbb{I} & 0 \\
0 & -\mathbb{I}
\end{array}\right), \alpha_{i}=\left(\begin{array}{cc}
0 & \sigma_{i} \\
\sigma_{i} & 0
\end{array}\right) \\
\sigma_{1}=\left(\begin{array}{ll}
0 & 1 \\
1 & 0
\end{array}\right), \quad \sigma_{2}=\left(\begin{array}{cc}
0 & -i \\
i & 0
\end{array}\right), \quad \sigma_{3}=\left(\begin{array}{cc}
1 & 0 \\
0 & -1
\end{array}\right), \\
\boldsymbol{p}=-i \nabla_{i} \text { and } \\
\mathcal{U}=\Lambda_{i}^{+} \Lambda_{j}^{+} V\left(\left|\boldsymbol{r}_{i}-\boldsymbol{r}_{j}\right|\right) \Lambda_{i}^{+} \Lambda_{j}^{+}
\end{gathered}
$$

where $\Lambda_{i}^{+}$is the positive spectral projection operator of a one-particle Hamiltonian similar to $h_{D}\left(r_{i}\right)$ [i.e. $\Lambda_{i}^{+} \phi=\phi$ for all eigenfunctions $\phi$ of $h_{D}\left(r_{i}\right)$ corresponding to positive eigenvalues]. Usually the potential used in this Hamiltonian is the direct Dirac-Fock potential (see Sec. 3). Moreover,

$$
\begin{aligned}
V\left(\left|\boldsymbol{r}_{i}-\boldsymbol{r}_{j}\right|\right) & =\frac{1}{r_{i j}}-\frac{\boldsymbol{\alpha}_{i} \cdot \boldsymbol{\alpha}_{j}}{r_{i j}}+\left(\frac{1}{r_{i j}}-\frac{\boldsymbol{\alpha}_{i} \cdot \boldsymbol{\alpha}_{j}}{r_{i j}}\right)\left(\cos \left(\omega_{i j} r_{i j} / c\right)-1\right) \\
& +c^{2}\left(\boldsymbol{\alpha}_{i} \cdot \nabla_{i}\right)\left(\boldsymbol{\alpha}_{j} \cdot \nabla_{j}\right) \frac{\cos \left(\omega_{i j} r_{i j} / c\right)-1}{\omega_{i j}^{2} r_{i j}}
\end{aligned}
$$

is the electron-electron interaction of order 1 in $\alpha=1 / c \approx 1 / 137$, the fine structure constant. This expression is in Coulomb gauge, and is derived directly from QED. Here $r_{i j}=\left|\boldsymbol{r}_{i}-\boldsymbol{r}_{j}\right|$ is the inter-electronic distance, $\omega_{i j}$ is the energy of the photon exchanged between the electron $i$ and $j$, which usually reduces to $\epsilon_{i}-\epsilon_{j}$ where the $\epsilon_{i}$ are the one-electron energies in the problem under consideration (for exemple diagonal Lagrange multipliers in the case of Dirac-Fock). Note that in (1.6) gradient operators act only on the $r_{i j}$ and not on the following wave functions. The presence of the $\omega_{i j}$ in this expression originates from the multi-time nature of the relativistic problem due to the finiteness of the speed of light. From this interaction, one can deduce the Breit operator, that contains retardation only to second order in $1 / c$, in which the $\omega_{i j}$ can be eliminated by use of commutation relations between $r$ and the one-particle Dirac Hamiltonian. This operator can then be readily used in the evaluation of correlation, while the higher-order in $1 / c$ in the interaction (1.6) can only be evaluated perturbatively.

Finding bound states of (1.2) is difficult and requires approximations. The different methods of solution are inspired from the non-relativistic problem. The three main categories of methods are the Relativistic Many-Body perturbation 
theory (RMBPT, see, e.g., [41] for the non-relativistic case), the Relativistic Random Phase Approximation (RRPA, see, e.g., [33]), which has been heavily used for evaluation of photoionization cross-sections, and Multiconfiguration Dirac-Fock (MCDF). The RMBPT method requires the use of basis sets to sum over intermediate states. The MCDF method is a variational method.

\section{Relativistic Many-Body perturbation theory and RRPA}

In its most general version, the RMBPT method starts from a multidimentional model space and uses Rayleigh-Schrödinger perturbation theory. The concept of model space is mandatory if there are several levels of quasidegenerate energy as in the ground state of Be-like ions $\left(1 s^{2} 2 s^{2}{ }^{1} S_{0}\right.$ and $1 s^{2} 2 p^{2}{ }^{1} S_{0}$ are very close in energy, leading to very strong intra-shell correlation). In that case one gets would get very bad convergence of the perturbation expansion, because of the near-zero energy denominators, if building the perturbation theory on a single level.

Following [41] we separate the Hamiltonian in a sum

$$
H_{T}=H_{0}+V_{0}
$$

We assume that we know a set of $N$ eigenfunctions $\Psi_{\alpha}^{0}$ of eigenenergies $E_{\alpha}^{0}$ which are all the solutions obtained by diagonalizing $H_{0}$ on a subspace $\mathcal{P}$ (these solutions can be obtained with the Dirac-Fock method in a suitable average potential). The unperturbed Hamiltonian is then chosen as

$$
H_{0}^{N}=P_{0} H_{0} P_{0}=\sum_{\alpha=1}^{N} E_{\alpha}^{0}\left|\Psi_{\alpha}^{0}\right\rangle\left\langle\Psi_{\alpha}^{0}\right|
$$

where and $P_{0}$ is the projector on $\mathcal{P}$, defined by

$$
P_{0}=\sum_{\alpha=1}^{N}\left|\Psi_{\alpha}^{0}\right\rangle\left\langle\Psi_{\alpha}^{0}\right| \text {. }
$$

We define the perturbation potential by

$$
V=H_{T}-P_{0} H_{0} P_{0}=H_{T}-H_{0}^{N}
$$

We also define $Q_{0}=1-P_{0}$ as the projector on the orthogonal space $\mathcal{Q}$. We now define the wave operator, which build the exact solution of the Hamiltonian equation (2.1) from the $\Psi_{\alpha}^{0}$

$$
\Psi_{\alpha}=\Omega \Psi_{\alpha}^{0}
$$


so that

$$
H_{T} \Psi_{\alpha}=E_{\alpha} \Psi_{\alpha}
$$

with the property

$$
P_{0} \Omega P_{0}=P_{0}
$$

The exact eigenenergies can be obtained by the application of the Model-space wave functions on the effective Hamiltonian

$$
H_{\text {eff }}=P_{0} H_{T} \Omega P_{0}=P_{0} H_{0} P_{0}+P_{0} V \Omega P_{0}=H_{0}^{N}+P_{0} V \Omega P_{0},
$$

using Eqs. (2.2) and (2.7), $P_{0}^{2}=P_{0}$ and the fact that $H_{0}^{N}$ and $P_{0}$ commute. This operator, acting on the unperturbed wave functions give the exact eigenenergies:

$$
H_{\mathrm{eff}} \Psi_{\alpha}^{0}=E_{\alpha} \Psi_{\alpha}^{0}
$$

The wave operator obeys the generalized Bloch equation

$$
\left[\Omega, H_{0}\right] P_{0}=V \Omega P_{0}-\Omega P_{0} V \Omega P_{0}
$$

using Eq. (2.7). This can be expanded in a series

$$
\Omega=1+\Omega^{(1)}+\Omega^{(2)}+\cdots
$$

Equations (2.10) and (2.11) leads to the sequence of equations

$$
\begin{aligned}
& {\left[\Omega^{(1)}, H_{0}\right] P_{0}=Q_{0} V P_{0}} \\
& {\left[\Omega^{(2)}, H_{0}\right] P_{0}=Q_{0} V \Omega^{(1)} P_{0}-\Omega^{(1)} P_{0} V P_{0}}
\end{aligned}
$$

The RRPA method is based on the solution of the Hamiltonian (1.2) subjected to a time-dependant perturbation (like a classical electromagnetic radiation of known frequency). This time-dependant Dirac-Fock equation is solved over a set of solutions of the unperturbed problem, leading to a set of time-dependant mixing coefficients in the usual fashion of time-dependant perturbation theory. The phases of those coefficients are approximated (leading to the name "Random Phase"), leading to differential equations very similar to the Dirac-Fock ones. This method include to all orders some classes of correlation contribution that can be easily also evaluated in the framework of RMBPT. It is mostly used for the ground state of atoms and ions to study photoionization. It is more difficult to use for excited states.

This paper is mostly devoted to the MCDF method for atoms and molecules, and to preliminary results for the linear Dirac operator. 


\section{The MCDF wave function}

We first start by describing shortly the formalism used to build the Dirac-Fock solutions for a spherically-symmetric system like an isolated atom.

If we define the angular momentum operators $\boldsymbol{L}=\boldsymbol{r} \wedge \boldsymbol{p}, \boldsymbol{J}=\boldsymbol{L}+\frac{\boldsymbol{\sigma}}{2}$, the parity $\Pi$ as $\beta P$, then the total wave function is expressed in term of configuration state functions (CSF) as antisymmetric products of one-electron wave functions so that they are eigenvalues of the parity $\Pi$, the total angular momentum $J$ and its projection $M$. The label $\nu$ stands for all other values (angular momentum recoupling scheme, seniority numbers, ...) necessary to define unambiguously the CSF. For a $N$-electron system, a CSF is thus a linear combination of Slater determinants:

$$
\left|\nu \prod J M>=\sum_{i=1} d_{i}^{\nu}\right| \begin{array}{ccc}
\Phi_{1}^{i, \nu}\left(r_{1}\right) & \cdots & \Phi_{N}^{i, \nu}\left(r_{1}\right) \\
\vdots & \ddots & \vdots \\
\Phi_{1}^{i, \nu}\left(r_{N}\right) & \cdots & \Phi_{N}^{i, \nu}\left(r_{N}\right)
\end{array} \mid
$$

all of them with the same $\Pi$ and $M$ values while the $d_{i}$ 's are determined by the requirement that the CSF is an eigenstate of $J^{2}$.

The total MCDF wave function is constructed as a superposition of CSF's, i.e.

$$
\Psi(\Pi J M)=\sum_{\nu=1}^{N C F} c_{\nu} \mid \nu \Pi J M>,
$$

where $N C F$ is the number of configurations and the $c_{\nu}$ are called the configurations mixing coefficients.

The MCDF method has two variants. In one variant, one uses numerical or analytic basis sets to construct the CSF. In the other one, direct numerical solution of the MCDF equation is used. Both methods have been used in atomic and molecular physics. The numerical MCDF method is better suited for small systems, while analytic basis set techniques are better suited for cases with millions of determinants.

This chapter is organized as follows. In Sec. 2, different choices of basis sets for the Dirac equation are presented. In Sec. 3, the MCDF equations are presented, and numerical techniques adapted to the numerical MCDF method in atoms are described. In Sec. 4, we deal with techniques for the numerical MCDF method in molecules. 


\section{Chapter 2. Linear Dirac equations}

\section{Properties of the linear Dirac operator}

The unboundedness from below of the Dirac operator

$$
H_{0}=-i c \alpha \cdot \nabla+m c^{2} \beta
$$

creates important difficulties when trying to find its eigenvalues. The so-called variational collapse is indeed related to this unboundedness property. On the other hand, finite dimensional approximations to this problem may lead to finding spurious solutions: some eigenvalues of the finite dimensional problem do not approach the eigenvalues of the Dirac operator and destroy the monotonicity of the approximated eigenvalues with respect to the basis dimension. These problems seem to be much more acute in molecular than in atomic computations, but they are already present in one-electron systems. In this section we address this difficulty for one-electron systems by describing various methods used to deal with this problem. Well-behaved approximation methods should also provide good nonrelativistic limits, that is, variational problems whose eigenvalues and eigenfunctions converge well to those of the corresponding nonrelativistic Schrödinger Hamiltonian.

A way often used to find good numerical approximations of eigenvalues of an operator $A$ consists in projecting the eigenvalue equation

$$
A x=\lambda x
$$

over a well chosen finite dimensional space $X_{N}$ of dimension $N$, in order to find an approximation $\left(\lambda_{N}, x_{N}\right)$ satisfying

$$
A_{N} x_{N}=\lambda_{N} x_{N}
$$

such that $\left(\lambda_{N}, x_{N}\right)$ converges to $(\lambda, x)$ as $N \rightarrow \infty$. Then one looks for the eigenvalues of the $N \times N$ matrix $A_{N}$ and these eigenvalues will converge either to eigenvalues of $A$ or to points in the essential spectrum of $A$. As $N$ increases, the limit set of the eigenvalues of $A_{N}$ is the spectrum of $A$.

The difficulty with the Dirac operator is that for most physically interesting potentials $V$, the spectrum of $H_{0}+V$ is made of its essential spectrum $\left(-\infty,-m c^{2}\right] \cup\left[m c^{2},+\infty\right)$ and a discrete set of eigenvalues lying in the gap $\left(-m c^{2}, m c^{2}\right)$. Hence, the choice of the finite dimensional space, or equivalently, of the finite basis set, is fundamental if we want to ensure that for some $N$ large, the eigenvalues of $\left(H_{0}+V\right)_{N}$, or at least some of them, will be approximations of the eigenvalues of $H_{0}+V$ in the gap $\left(-m c^{2}, m c^{2}\right)$. The 
question of how to choose a good basis set has been addressed in many papers, among which $[11,12,22,21,32,35,38]$, that we will describe with further details in Sections 5 and 6 below. In particular, Section 6 is devoted to the description of numerical techniques based either on discretization or on BSplines, and shows that with appropriate boundary conditions one can avoid the variational collapse.

When the operator $A$ is bounded from below, it is often possible to characterize its spectrum by variational methods, for instance by looking for critical values of the Rayleigh quotient

$$
Q(x):=\frac{(A x, x)}{(x, x)}
$$

over the domain of $A$. More concretely, when $A$ is bounded from below, under appropriate assumptions, its ground state energy can be found by minimizing the above Rayleigh quotient. However, this cannot be done directly in the context of the Dirac operator, since it is unbounded from below (and also from above). A large number of works have been devoted to the variational resolution of this problem in view of the Dirac operator. Most of them use the approximation of an effective Hamiltonian which is bounded from below. The idea hidden behind this kind of techniques is that there is no explicit way of diagonalizing the Dirac Hamiltonian $H+V$, but this can be done at an abstract level. The diagonalized operator is then approximated via a finite expansion or an iterative procedure. These methods are therefore perturbative and contain an approximation at the operator level. They will be referred to as perturbation theories and effective Hamiltonian methods and will be described below (see [11,35,21,32] and Section 7 for more details).

Other variational techniques are based on a correspondence between the eigenvalues of $A$ and those of $T(A)$, for some operator function $T$, like the inverse function $T x=x^{-1}$ (see [29]) or the function $T x=x^{2}$ (see [56,1]). Finally, some authors solve the variational problem in a subspace of the domain in which the operator is bounded from below and 'avoids' the negative continuum. Section $\nmid 8$ will be devoted to these more direct variational approaches, based on either linear or nonlinear constraints.

Before going into the details of the computational methods, let us start with some notations and preliminary considerations. For any $\psi$ with values in $\mathbb{C}^{4}$, if we write $\psi=\left(\begin{array}{l}\varphi \\ \chi\end{array}\right)$, with $\varphi, \chi$ taking values in $\mathbb{C}^{2}$, then the eigenvalue equation

$$
H \psi=\left(H_{0}+V\right) \psi=\lambda \psi
$$


is equivalent to the following system:

$$
\left\{\begin{array}{l}
R \chi=\left(\lambda-m c^{2}-V\right) \varphi \\
R \varphi=\left(\lambda+m c^{2}-V\right) \chi
\end{array}\right.
$$

with $R=i c(\vec{\sigma} \cdot \vec{\nabla})=\sum_{j=1}^{3} i c \sigma_{j} \frac{\partial}{\partial x_{j}}$. Here $\sigma_{j}, j=1,2,3$, are the Pauli matrices. As long as $\lambda+m c^{2}-V \neq 0$, the system (4.6) can be written as

$$
H^{\mu} \varphi:=R\left(\frac{R \varphi}{g_{\mu}}\right)+V \varphi=\mu \varphi, \quad \chi=\frac{R \varphi}{g_{\mu}}
$$

where $g_{\mu}=\mu+2 m c^{2}-V$ and $\mu=\lambda-m c^{2}$. Note that the Hamiltonian operator $H^{\mu}$ is eigenvalue dependent. Reducing the 4-component spinor $\psi$ to an equation for the 2-spinor $\varphi$ is often called partitioning. Let us immediately notice that at least formally, the partitioned equation (4.7) converges to its nonrelativistic counterpart

$$
-\frac{1}{2 m} \Delta \varphi+V \varphi=\mu \varphi
$$

(see for instance [58]). For this reason, but also because the principal part of the second order operator in (4.7) is semibounded for not too large potentials $V$, the partitioned equation has been extensively studied for finding eigenvalues of linear Dirac operators.

To end these preliminary considerations on linear Dirac equations, note that in the case of rotationally invariant potentials, the solutions can be put in the form

$$
\psi=\frac{1}{r}\left(\begin{array}{c}
P_{\kappa}(r) \chi_{\kappa m}(\theta, \varphi) \\
i Q_{\kappa}(r) \chi_{-\kappa m}(\theta, \varphi)
\end{array}\right)
$$

The dependence on the angular coordinates is contained in the 2-spinors $\chi_{ \pm \kappa m}(\theta, \varphi)$, which are eigenfunctions of the angular momentum operators $J$, its third component $J_{z}$ (with eigenvalues $j(j+1)$ and $m$ respectively) and of parity. On the other hand, the radial dependence is contained in the functions $f$ and $g$ which are called the upper and lower radial components of $\psi$.

In the ansatz defined in (4.9), for a given $\kappa= \pm\left(j+\frac{1}{2}\right)$, with $j=\ell \mp \frac{1}{2}$, $l=0,1, \ldots$, the eigenvalue equation $(4.5)$ is equivalent to

$$
\left(H_{r}^{\kappa}+V\right) \Phi=\lambda \Phi
$$




$$
\text { with } \quad H_{r}^{\kappa}=\left(\begin{array}{cc}
m c^{2} & c\left(-\frac{d}{d r}+\kappa_{j}\right) \\
c\left(\frac{d}{d r}+\kappa_{j}\right) & -m c^{2}
\end{array}\right)
$$

$\Phi=\left(\begin{array}{l}P_{\kappa} \\ Q_{\kappa}\end{array}\right)$ being a 2-vector with two scalar real components.

\section{$5 \quad$ Finite basis set approaches}

The choice of finite dimensional spaces is essential for the discretization of the operator and the approximation of its eigenvalues. The presence of the negative continuum makes this task difficult in the case of the Dirac operator. The basic criterium to decide whether a particular space, or a generating basis set, is good, is to check that the approximated eigenvalues found are either negative and lying in the negative continuum or positive. In this case, if they are below the positive continuum, they are approximations of the discrete exact (positive) eigenvalues. Many attempts to construct finite basis sets can be found in the litterature.

In [11], Drake and Goldman introduced the so-called Slater type orbitals (STO):

$$
\Phi(r)=r^{\gamma-1} e^{-\nu r} \sum_{i=1}^{N} r^{i}\left[a_{i}\left(\begin{array}{l}
1 \\
0
\end{array}\right)+b_{i}\left(\begin{array}{l}
0 \\
1
\end{array}\right)\right]
$$

with a particular choice of $\gamma$ and $\nu$ which depends on $\kappa$ and $V$. They showed numerical evidence that such a finite basis satisfies the above properties in the case of hydrogen-like atoms. Note that the STOs exhibit the same behavior near 0 and at $\infty$ as the exact eigenfunctions. The properties of STO basis sets are made more explicit in [21], where STO basis sets are replaced by orthonormal sets of Laguerre polynomials. The main drawback of this approach is that some of the eigenvalues of the approximated matrix are spurious roots which do not approximate any of the exact eigenvalues.

Another way to construct basis sets with good properties consists in imposing the so-called kinetic balance condition relating the upper and lower components of the functions in the basis set. See for instance [35].

Other types of basis sets proposed in the litterature include those generated by $B$-splines (see [32]), which have very good properties since, in this approach, the matrices are very sparse: only a finite number (depending on the degree of the splines) of diagonal lines are nonzero. This kind of basis sets has been widely used in atomic and molecular computations (see Sec. 6). 
The choice of a good basis set can be quite effective in some computations, but as it appears clearly in the litterature that we quote, there is very often a risk of finding spurious roots or of variational collapse. In the next subsection we give some more precise exemples of how to use particular basis sets in the context of Dirac operators.

\section{$6 \quad$ Numerical basis sets}

This section is devoted to the special case of basis sets whose elements are computed numerically.

\section{Discretisation method}

The Göteborg group has developed an efficient technique to obtain basis sets for the Dirac equation [47]. The Dirac equation is discretized and solved on a grid. The atom is placed in a spherical box, large enough not to disturb the bound state wave function considered. The method provides a finite number of orbitals which is complete over the discretized space [48], and resemble lattice gauge field calculation [57]. The method enables to eliminate spurious states and preserves the Hermiticity of the discretized Hamiltonian. The appearance of spurious states in a discretized method, is traced back to the "fermion doubling", first encoutered in gauge-field lattice calculations [34]. On a lattice of dimension $(D+1)$ ( $D$ spatial and one time dimensions), an equation for a massless fermion will describe not one but $2^{D}$ ones if no precaution is taken [51].

As an example, let us consider a one-dimensional Dirac equation for a free fermion

$$
\left(\begin{array}{c}
m c^{2}-c \frac{d}{d x} \\
c \frac{d}{d x}-m c^{2}
\end{array}\right)\left(\begin{array}{l}
f(x) \\
g(x)
\end{array}\right)=\left(\epsilon+m c^{2}\right)\left(\begin{array}{l}
f(x) \\
g(x)
\end{array}\right)
$$

The derivatives are approximated over the latice points using

$$
f_{i}^{\prime}=\frac{f_{i+1}-f_{i-1}}{2 h}
$$

where $h$ is the space between adjacent lattice sites. Eliminating the large component in (6.1), one gets the following equation

$$
-\frac{1}{2 m}\left(\frac{f_{i+2}-2 f_{i}+f_{i-2}}{4 h^{2}}\right)=\epsilon\left(1+\frac{\epsilon}{2 m c^{2}}\right) f_{i},
$$

in which the left hand side is the kinetic energy operator $p_{x}^{2} / 2 m$ acting on $f$ at the latice point $i$. Yet this second order derivative does not connect even 
and odd lattice sites. The highest energy solution over the lattice is the one changing sign at each site so that

$$
\cdots \approx f_{i-2} \approx-f_{i-1} \approx f_{i} \approx-f_{i+1} \approx \cdots
$$

Using the expression (6.3) acting on this solution gives the same results as if it had no nodes. A high-energy eigenvector thus appears as a spurious state in the low energy part of the spectrum. For low-order derivative two equivalent ways can be used $[51,47]$. One is to use forward derivatives for $f$ and backward derivatives for $g$,

$$
f_{i}^{\prime}=\frac{f_{i+1}-f_{i}}{h}, \quad g_{i}^{\prime}=\frac{g_{i}-g_{i-1}}{h} .
$$

The other consists in defining the large and small components on alternating sites on the lattice.

$$
\cdots f_{i-3} g_{i-2} f_{i-1} g_{i} f_{i+1} g_{i+2} \cdots
$$

with $h$ being the separation between $g_{i-2}$ and $g_{i}$. In this case the derivative is expressed as

$$
f_{i}^{\prime}=\frac{f_{i+1}-f_{i}}{h}, \quad g_{j}^{\prime}=\frac{g_{j}-g_{j-1}}{h} .
$$

with $i=2 n-1, j=2 n, n=1,2, \ldots, N$. These methods reduce to the same second-order equation [51].

Salomonson and Öster use a more accurate six-point formula

$$
\begin{aligned}
f^{\prime}(x)=\frac{1}{1920 h}[ & -9 f\left(x-\frac{5}{2} h\right)+125 f\left(x-\frac{3}{2} h\right)-2250 f\left(x-\frac{1}{2} h\right) \\
& +2250 f\left(x+\frac{1}{2} h\right)-125 f\left(x+\frac{3}{2} h\right) \\
& \left.+9 f\left(x+\frac{5}{2} h\right)\right]+\mathcal{O}\left(h^{6}\right) .
\end{aligned}
$$

This six-point formula combined with (6.6) provides a spurious-state-free solution, while using the same lattice for $f$ and $g$ and a forward-backward derivative scheme does not work.

In the spherical case, one needs to use a logarithmic lattice to get a good description of the wave function. The Hermiticity of the Hamiltonian must be preserved by doing the variable change

$$
y(r) \rightarrow \frac{1}{\sqrt{r}} y(x), \quad x=\log (r) .
$$


The corresponding Dirac equation is

$$
\left(\begin{array}{cc}
V(r) & -c\left(\frac{1}{\sqrt{r}} \frac{d}{d x} \frac{1}{\sqrt{r}}-\frac{\kappa}{\sqrt{r}} \frac{1}{\sqrt{r}}\right) \\
c\left(\frac{1}{\sqrt{r}} \frac{d}{d x} \frac{1}{\sqrt{r}}+\frac{\kappa}{\sqrt{r}} \frac{1}{\sqrt{r}}\right) & V(r)-m c^{2}
\end{array}\right)\left(\begin{array}{l}
f(x) \\
g(x)
\end{array}\right)=\epsilon\left(\begin{array}{l}
f(x) \\
g(x)
\end{array}\right) .
$$

Since the large and small component are defined on different lattices, one needs interpolation formulas to express $f(x) / \sqrt{r}$ and $g(x) / \sqrt{r}$ in the $\kappa$ term.

The discretization finally provides a $2 N \times 2 N$ symmetric eigenvalue problem

$$
\left(\begin{array}{cc}
A & { }^{t} D+{ }^{t} K \\
D+K & B
\end{array}\right)\left(\begin{array}{l}
F \\
G
\end{array}\right)=\epsilon\left(\begin{array}{l}
F \\
G
\end{array}\right)
$$

with $(F, G)=\left(f_{1}, f_{3}, \ldots, f_{2 N-1}, g_{2}, g_{4}, \ldots, g_{2 N}\right)$. For a point nucleus, the submatrices are $A_{i i}=-Z / r_{i}$ and $B_{j j}=-2 m c^{2}-Z / r_{j}, i=2 n-1, j=2 n$, $n=1,2, \ldots, N$. With the 6 points interpollation and derivation formulas used in [47], one obtains

$$
D=\frac{c}{1920 h}\left(\begin{array}{ccccccc}
-\frac{2250}{\sqrt{r_{2} r_{1}}} & \frac{2250}{\sqrt{r_{2} r_{3}}} & -\frac{125}{\sqrt{r_{2} r_{5}}} & \frac{9}{\sqrt{r_{2} r_{7}}} & 0 & \ldots & \ldots \\
\frac{125}{\sqrt{r_{4} r_{1}}} & -\frac{2250}{\sqrt{r_{4} r_{3}}} & \frac{2250}{\sqrt{r_{4} r_{5}}} & -\frac{125}{\sqrt{r_{4} r_{7}}} & \frac{9}{\sqrt{r_{4} r_{9}}} & 0 & \ldots \\
-\frac{9}{\sqrt{r_{6} r_{1}}} & \frac{125}{\sqrt{r_{6} r_{3}}} & -\frac{2250}{\sqrt{r_{6} r_{5}}} & \frac{2250}{\sqrt{r_{6} r_{7}}} & -\frac{125}{\sqrt{r_{6} r_{9}}} & \frac{9}{\sqrt{r_{6} r_{11}}} & \cdots \\
0 & -\frac{9}{\sqrt{r_{8} r_{3}}} & \frac{125}{\sqrt{r_{8} r_{5}}} & -\frac{2250}{\sqrt{r_{8} r_{7}}} & \frac{2250}{\sqrt{r_{8} r_{9}}} & -\frac{125}{\sqrt{r_{8} r_{11}}} & \cdots \\
\vdots & & \vdots & \vdots & \vdots & \vdots & \ddots
\end{array}\right),
$$

and

$$
K=\frac{\kappa}{256 h}\left(\begin{array}{ccccccc}
\frac{150}{\sqrt{r_{2} r_{1}}} & \frac{150}{\sqrt{r_{2} r_{3}}} & -\frac{25}{\sqrt{r_{2} r_{5}}} & \frac{3}{\sqrt{r_{2} r_{7}}} & 0 & \ldots & \ldots \\
-\frac{25}{\sqrt{r_{4} r_{1}}} & \frac{150}{\sqrt{r_{4} r_{3}}} & \frac{150}{\sqrt{r_{4} r_{5}}} & -\frac{25}{\sqrt{r_{4} r_{7}}} & \frac{3}{\sqrt{r_{4} r_{9}}} & 0 & \ldots \\
\frac{3}{\sqrt{r_{6} r_{1}}} & -\frac{25}{\sqrt{r_{6} r_{3}}} & \frac{150}{\sqrt{r_{6} r_{5}}} & \frac{150}{\sqrt{r_{6} r_{7}}} & -\frac{25}{\sqrt{r_{6} r_{9}}} & \frac{3}{\sqrt{r_{6} r_{11}}} & \ldots \\
0 & \frac{3}{\sqrt{r_{8} r_{3}}} & -\frac{25}{\sqrt{r_{8} r_{5}}} & \frac{150}{\sqrt{r_{8} r_{7}}} & \frac{150}{\sqrt{r_{8} r_{9}}} & -\frac{25}{\sqrt{r_{8} r_{11}}} & \ldots \\
\vdots & & \vdots & \vdots & \vdots & \vdots & \ddots
\end{array}\right) .
$$

Equation (6.11) is symmetric even though $K$ and $D$ are not. In the upper left corner of $\mathrm{D}$, use has been made of the approximation

$$
f(r) \sim r^{\gamma+1 / 2}+\frac{2\left[\gamma+\kappa-(Z \alpha)^{2}\right]}{Z \alpha^{2}(2 \gamma+1)} r^{\gamma+3 / 2}+\epsilon \frac{\left[\gamma+\kappa-2(Z \alpha)^{2}\right]}{Z(2 \gamma+1)} r^{\gamma+3 / 2}
$$

with $\gamma=\sqrt{\kappa^{2}-(Z \alpha)^{2}}$, and the equivalent expression for $g$. To avoid nonlinear terms in the eigensystem (6.11), only the contribution independent of 
$\epsilon$ has been kept. This is a good approximation for bound states for which $\epsilon \ll m c^{2}$.

\section{Numerical basis sets based on B-splines}

$B$-splines have been used [32] to provide numerically efficient basis sets. A knot sequence $t_{i}$ is used for the radial coordinate, on which B-spline of order $k$ provide a complete basis for piecewise polynomials of order $k-1$. This radial coordinate extends to a distance $R$ from the origin. The solutions of the Dirac equation are expressed as linear combinations of $B$-splines. A Galerkin method is employed to obtain the solution. The Dirac equation is derived from an action principle $\delta S=0$, with

$$
\begin{aligned}
S= & \frac{1}{2} \int_{0}^{R}\left\{c P_{\kappa}(r)\left(\frac{d}{d r}-\frac{\kappa}{r}\right) Q_{\kappa}(r)-c Q_{\kappa}(r)\left(\frac{d}{d r}+\frac{\kappa}{r}\right) P_{\kappa}(r)\right. \\
& \left.+V_{N}(r)\left[P_{\kappa}(r)^{2}+Q_{\kappa}(r)^{2}\right]-2 m c^{2} Q_{\kappa}(r)^{2}\right\} d r \\
& -\frac{1}{2} \epsilon \int_{0}^{R}\left[P_{\kappa}(r)^{2}+Q_{\kappa}(r)^{2}\right] d r
\end{aligned}
$$

using the notations of (4.9) (note that in this representation the gap lies between $-2 m c^{2}$ and 0 ), to which suitable boundary conditions are added through

$$
S^{\prime}=\left\{\begin{array}{l}
\frac{c}{4}\left[P_{\kappa}(R)^{2}-Q_{\kappa}(R)^{2}\right]+\frac{c}{2} P_{\kappa}(0)^{2}-\frac{c^{2}}{2} P_{\kappa}(0) Q_{\kappa}(0) \text { for } \kappa<0, \\
\frac{c}{4}\left[P_{\kappa}(R)^{2}-Q_{\kappa}(R)^{2}\right]+c^{2} P_{\kappa}(0)^{2}-\frac{c}{2} P_{\kappa}(0) Q_{\kappa}(0) \text { for } \kappa>0 .
\end{array}\right.
$$

From the point of view of the variational principle, $\epsilon$ is a Lagrange multiplier introduced to ensure that the solutions of the Dirac equation are normalized. The boundary constraint (6.16) is designed to avoid a hard boundary at the box radius $R$, following the idea behind the MIT bag model for quark confinement, and provides $P_{\kappa}(R)=Q_{\kappa}(R)$. Forcing $P_{\kappa}(R)=Q_{\kappa}(R)=0$ would amount to introduce an infinite potential at the boundary and possibly leads to the Klein paradox. Other choices of boundary conditions are possible. This particular choice avoids the appearance of spurious solutions. Expanding the radial wave function as

$$
P_{\kappa}(r)=\sum_{i=1}^{n} p_{i} B_{i, k}(r), \quad Q_{\kappa}(r)=\sum_{i=1}^{n} q_{i} B_{i, k}(r),
$$

the variational principle reduces to

$$
\frac{d\left(S+S^{\prime}\right)}{d p_{i}}=0, \quad \frac{d\left(S+S^{\prime}\right)}{d q_{i}}=0, \quad i=1,2, \ldots, n .
$$

This leads to a $2 n \times 2 n$ symmetric, generalized eigenvalue equation 


$$
A v=\epsilon B v
$$

where $v=\left(p_{1}, p_{2}, \ldots, p_{n}, q_{1}, q_{2}, \ldots, q_{n}\right)$,

$$
A=\left(\begin{array}{cc}
(V) & c\left[(D)-\left(\frac{\kappa}{r}\right)\right] \\
-c\left[(D)+\left(\frac{\kappa}{r}\right)\right] & (V)-2 m c^{2}(C)
\end{array}\right)+A^{\prime}
$$

and

$$
B=\left(\begin{array}{cc}
(C) & 0 \\
0 & (C)
\end{array}\right)
$$

The $2 n \times 2 n$ matrix $A^{\prime}$ comes from the boundary term. The $n \times n$ matrix $(C)$ is the B-spline overlap matrix defined by

$$
(C)_{i j}=\int B_{i, k}(r) B_{j, k}(r) d r
$$

(D) comes from the differential operator

$$
(D)_{i j}=\int B_{i, k}(r) \frac{d B_{j, k}(r)}{d r} d r
$$

$(V)$ is the potential term

$$
(V)_{i j}=\int B_{i, k}(r) V_{N}(r) B_{j, k}(r) d r \quad \text { and } \quad\left(\frac{\kappa}{r}\right)_{i j}=\int B_{i, k}(r) \frac{\kappa}{r} B_{j, k}(r) d r .
$$

Diagonalization of (6.20) provides $2 n$ eigenvalues and eigenfunctions, $n$ of which have energies below $-2 m c^{2}$, a few correspond to bound states (typically 5 to 6 for $k=7$ to 9 ) and the rest belongs to the positive energy continuum.

\section{Perturbation theory and effective Hamiltonians}

An alternative way to find the eigenvalues of the unbounded relativistic operator $H$ consists in looking for a so-called effective Hamiltonian $H^{\text {eff }}$, which is semi-bounded, such that both Hamiltonians have common eigenvalues on an interval above the negative continuous spectrum. Such a Hamiltonian $H^{\text {eff }}$ cannot usually be found in an explicit way, but can be viewed as the limit of an iterative procedure. This leads to families of Hamiltonians which approach the effective Hamiltonian and yield approximated eigenvalues for $H$. 
One of the most popular procedure in this direction is due to Foldy and Wouthuysen [19], whose main idea was to apply a unitary transformation $\Omega$ to $H_{0}+V$ such that

$$
\Omega^{*}\left(H_{0}+V\right) \Omega=H^{F W}=\left(\begin{array}{cc}
H_{+}^{F W} & 0 \\
O & H_{-}^{F W}
\end{array}\right)
$$

so that electronic and positronic states are decoupled: electrons (resp. positrons) would be described by the eigenfunctions of $H_{+}^{F W}$ (resp. $H_{-}^{F W}$ ). Moreover, the Hamiltonians $H_{+}^{F W}-m c^{2}$ (resp. $H_{-}^{F W}+m c^{2}$ ) are bounded from below (resp. above) and have correct nonrelativistic limits. Although this procedure looks very promising, the problem is that $\Omega$ is unknown in closed form, and so there is no way of diagonalizing $H_{0}+V$ in an explicit way. However, approximations of $\Omega$, and therefore of $H^{F W}$, can be constructed either by writing a formal series expansion for $H_{ \pm}^{F W}$ in the perturbation parameter $c^{-2}$ :

$$
H_{ \pm}^{F W}=\sum_{k=0}^{+\infty} c^{-2 k} H_{2 k}^{ \pm}
$$

and cutting it at level $k \geq 0$, or by approaching it by an iterative procedure.

In general one identifies the effective Hamiltonian $H^{\text {eff }}$ as a solution to a nonlinear equation $H^{\text {eff }}=f\left(H^{\text {eff }}\right)$, which can be solved approximately in an iterative way. By instance, one can produce an equation like the above one by "eliminating" the lower component $\chi$ of the spinor as in (4.7), that is, by partitioning.

Many proposals of effective Hamiltonians for the Dirac operator can be found in the litterature. Some are Hermitian, some are not, some act on 4 component spinors, others on 2-spinors. A good review about various approaches to this problem and the corresponding difficulties has been written by W. Kutzelnigg [37] (see also $[36,45,46]$ ). An important difficulty arising in this context is that most of the proposed effective Hamiltonians are quite nice when the potential $V$ is regular, but in the case of the Coulomb potential they contain very singular terms, which are not even well defined near the nucleus. These serious singularities are avoided by a method used by Chang, Pélissier and Durand [4] (see also [13,14]), where it is proposed to use $\left(2 m c^{2}-V\right)^{-1}$ as an expansion parameter in the formal series defining $H^{\text {eff }}$, instead of $c^{-2}$. They obtain a 2-component Pauli-like Hamiltonian which is bounded from below, contains only well defined terms and approaches $H$. Similar ideas have been used by Heully et al. [28] and by Van Lenthe et al. [39,40]. The latter have also made a systematic numerical analysis of this method in self-consistent calculations for the uranium atom. 


\section{Direct variational approaches}

To begin with, let us mention two variational methods based on nonlinear transformations of the Hamiltonian. Wallmeier and Kutzelnigg in [56] look for eigenvalues of the squared Hamiltonian $\left(H_{0}+V\right)^{2}$. The practical difficulty arises from the need to compute complicated matrix representations. Hill and Krautkauser [29] use the Rayleigh-Ritz variational principle applied to the inverse of the Dirac Hamiltonian, $1 / H$. A difficulty arises here in the computation of the matrix elements for the inverse operator. This is avoided by working in the special set of test functions defined by those which are in the image by $H$ of a regular set of spinors. The use of these two methods can be useful in some cases, but not when the eigenvalues become close to 0 .

As already noticed, the eigenvalues of the operator $H_{0}+V$ are critical points of the Rayleigh quotient

$$
Q_{V}(\psi):=\frac{\left(\left(H_{0}+V\right) \psi, \psi\right)}{(\psi, \psi)}
$$

in the domain of $H_{0}+V$. We are now going to describe other more sophisticated variational approaches yielding exact eigenvalues of $H_{0}+V$. The particular structure of the spectrum of $H_{0}$ clearly shows that eigenvalues of $H_{0}+V$ lying in the gap of the essential spectrum should be given by some kind of min-max approach. This had been mentioned in several papers dealing with numerical computations of Dirac eigenvalues, before it was proved in a series of papers: $[18,26,8,25,9])$. Basically, in all those papers, it was shown that under appropriate assumptions on the potential $V$, the eigenvalues are indeed characterized as a sequence of min-max values defined for $Q_{V}$ on well chosen sets. A theorem in [9] proves that for a large class of potentials $V$, the ground state energy of $H_{0}+V$ is given by the smallest $\lambda$ in the gap $\left[-m c^{2}, m c^{2}\right]$ such that there exists $\varphi$ satisfying

$$
\lambda \int_{\mathbb{R}^{3}}|\varphi|^{2} d x=\int_{\mathbb{R}^{3}}\left(\frac{|(\sigma \cdot \nabla) \varphi|^{2}}{1-V+\lambda}+(1+V)|\varphi|^{2}\right) d x
$$

and the corresponding eigenfunction is the spinor function

$$
\psi=\left(\begin{array}{c}
\varphi \\
-i \frac{(\sigma \cdot \nabla) \varphi}{1-V+\lambda}
\end{array}\right)
$$

Note that the idea to build a semibounded energy functional had already been introduced by Bayliss and Peel [1] in another context. It is closely related to previous works of Datta and Deviah [5], and Talman [54], where a particular min-max procedure for the Rayleigh quotient $Q_{V}$ is proposed without proof. We will not give here further details on these theoretical aspects (for tractable numerical applications, see below). 
An alternative variational method has been proposed by Dolbeault, Esteban and Séré in [8]. It is based on rigorous results proving that for a very large class of potentials (including all those relevant in atomic models), the ground state of $H_{0}+V$ can be found by a minimization problem posed in a class of functions defined by a nonlinear constraint. The main idea is to eliminate the lower component of the spinor and solve a minimization problem for the upper one. With the notations of the introduction, $\psi=\left(\begin{array}{l}\varphi \\ \chi\end{array}\right)$ is an eigenfunction of $H_{0}+V$ if and only if (4.7) takes place. The first equation in (4.7) is an elliptic second order equation for the upper component $\varphi$, while the second part of (4.7) gives the lower component $\chi$ as a function of $\varphi$ and the eigenvalue $\lambda$. The dependence of $H^{\mu}$ on $\lambda=\mu+m c^{2}$ makes this problem nonlinear, since $\lambda$ is still to be found, but the difficulty of finding the unitary transformation $\Omega$ in the Foldy-Wouthuysen approach is now replaced by a much simpler problem.

We may reformulate the question as follows. Let $A(\lambda)$ be the operator defined by the quadratic form acting on 2-spinors:

$$
\varphi \mapsto \int_{\mathbb{R}^{3}}\left(\frac{|(\sigma \cdot \nabla) \varphi|^{2}}{1-V+\lambda}+(1-\lambda+V)|\varphi|^{2}\right) d x=:(\varphi, A(\lambda) \varphi)
$$

and consider its lowest eigenvalue, $\mu_{1}(\lambda)$. Because of the monotonicity with respect to $\lambda$, there exists at most one $\lambda$ for which $\mu_{1}(\lambda)=0$. This $\lambda$ is the ground state level.

An algorithm to numerically solve the above problem has been proposed in [10]. The idea consists in discretizing Eq. (8.2) in a finite dimensional space $E_{n}$ of dimension $n$ of 2 -spinor functions. The discretized version of (8.4) is

$$
A^{n}(\lambda) x_{n} \cdot x_{n}=0
$$

where $x_{n} \in E_{n}$ and $A^{n}(\lambda)$ is a $\lambda$-dependent $n \times n$ matrix. If $E_{n}$ is generated by a basis set $\left\{\varphi_{i}, \ldots \varphi_{n}\right\}$, the entries of the matrix $A^{n}(\lambda)$ are the numbers

$$
\int_{\mathbb{R}^{3}}\left(\frac{\left((\sigma \cdot \nabla) \varphi_{i},(\sigma \cdot \nabla) \varphi_{j}\right)}{1-V+\lambda}+(1-\lambda+V)\left(\varphi_{i}, \varphi_{j}\right)\right) d x
$$

The ground state energy will then be approached from above by the unique $\lambda$ for which the first eigenvalue of $A^{n}(\lambda)$ is zero. This method has been tested on a basis of Hermite polynomials (see [10] for some numerical results). More efficient computations have been made recently on radially symmetric configurations with $B$-splines basis sets, involving very sparse matrices. Approximations from above of the excited levels can also be computed by requiring successively that the second, third,... eigenvalues of $A^{n}(\lambda)$ are equal to zero. 


\section{Chapter 3. The MCDF method for atoms}

\section{The Muticonfiguration Dirac-Fock (MCDF) method}

The MCDF equations are obtained from (1.2) by a variational principle. The energy functional is written

$$
E_{\mathrm{tot}}=\frac{<\nu \Pi J M\left|H^{\mathrm{np}}\right| \nu \Pi J M>}{<\nu \Pi J M|| \nu \Pi J M>}
$$

A Hamiltonian matrix which provides the mixing coefficients by diagonalization is obtained from (9.1) with the help of

$$
\frac{\partial}{\partial c_{\nu}} E_{\mathrm{tot}}=0
$$

and a set of integro-differential equations for the radial wave functions $P_{\kappa}(r)$ and $Q_{\kappa}(r)$ is obtained from the functional derivatives

$$
\left\{\begin{array}{l}
\frac{\delta}{\delta P_{\kappa}(r)} E_{\mathrm{tot}}=0 \\
\frac{\delta}{\delta Q_{\kappa}(r)} E_{\mathrm{tot}}=0
\end{array}\right.
$$

One assumes the orthogonality condition (restricted Dirac-Fock)

$$
\int_{0}^{\infty}\left[P_{A}(r) P_{B}(r)+Q_{A}(r) Q_{B}(r)\right] d r=\delta_{\kappa_{A}, \kappa_{B}} \delta_{n_{A}, n_{B}}
$$

in order to make the angular calculations possible. Equation (9.3) then leads to the inhomogeneous Dirac equation for a given orbital $A$

$$
\left(\begin{array}{cc}
\frac{d}{d r}+\frac{\kappa_{A}}{r} & -\frac{2}{\alpha}+\alpha V_{A}(r) \\
-\alpha V_{A}(r) & \frac{d}{d r}-\frac{\kappa_{A}}{r}
\end{array}\right)\left(\begin{array}{c}
P_{A}(r) \\
Q_{A}(r)
\end{array}\right)=\alpha \sum_{B} \epsilon_{A, B}\left(\begin{array}{c}
Q_{B}(r) \\
-P_{B}(r)
\end{array}\right)+\left(\begin{array}{c}
X_{Q_{A}}(r) \\
-X_{P_{A}}(r)
\end{array}\right)
$$

where $V_{A}$ is the sum of the nuclear potential and the direct Coulomb potential, while the exchange terms $X_{P_{A}}$ and $X_{Q_{A}}$ include all the two-electron interactions except for the direct Coulomb instantaneous repulsion. The constants $\epsilon_{A, B}$ are Lagrange parameters used to enforce the orthogonality constraints of (9.4) and thus the summation over $B$ runs only for orbitals with $\kappa_{B}=\kappa_{A}$. The exchange terms can be very large if the orbital A has a small effective occupation (the exchange term is a sum of exchange potentials divided by the 
effective occupation of the orbitals). This effective occupation is the sum

$$
o_{A}=\sum_{i=1}^{N C F} c_{\nu}^{2} q_{\nu}^{(A)}
$$

where $q_{\nu}^{(A)}$ is the number of electrons in the orbital $A$ in the $\nu$ th configuration.

The numerical MCDF methods are based on a fixed-point method, or to be precise on an iteration scheme which provides a self-consistent field (SCF) state in a way very similar to the method which is used to solve the HartreeFock model. Initial wave functions must be chosen, e.g., hydrogenic wave functions, wave functions in a Thomas-Fermi potential or wave functions already optimized with a smaller set of configurations. One then builds the Hamiltonian matrix (9.2) and obtains the mixing coefficients. Those coefficients and the initial wave functions enter the direct and exchange potential in (9.5), which become normal differential equations, and are solved numerically for each orbital. A new set of potential terms is then evaluated until all the wave functions are stable to a given accuracy $\left(\approx 10^{-2}\right.$ in the first cycle of diagonalization to $\approx 10^{-6}$ at the last cycle, at the point where the largest variation occurs). A new Hamiltonian matrix is then built and new mixing coefficients are calculated. This process is repeated until convergence is reached. As it is a highly nonlinear process, this can be very tricky, and trial and error on the initial conditions is often required when many configuration and correlation orbitals (i.e. orbitals with very small effective occupations) are involved. All those calculations are done using direct numerical solutions of the MCDF differential equations (9.5), which has the advantage of providing very accurate results with relatively limited set of configurations, while MCDF methods using basis set require orders of magnitude more configurations to achieve similar accuracies.

Explicit expressions for $V_{A}, X_{P_{A}}$ and $X_{Q_{A}}$ can be found in [23,24,6]. All potentials can be expressed in term of the functions

$$
\begin{gathered}
Z_{i, j}^{k}(x)=\frac{1}{x^{k}} \int_{0}^{x} d r \rho_{i j}(r) r^{k} \\
Y_{i, j}^{k}(x)=\frac{1}{x^{k}} \int_{0}^{x} d r \rho_{i j}(r) r^{k}+x^{k+1} \int_{x}^{\infty} d r \frac{\rho_{i j}(r)}{r^{k+1}},
\end{gathered}
$$

where $\rho_{i j}(r)=P_{i}(r) P_{j}(r)+Q_{i}(r) Q_{j}(r)$ for the Coulomb part of the interaction, to which are added terms with $\rho_{i j}(r)=P_{i}(r) Q_{j}(r)$ or $\rho_{i j}(r)=Q_{i}(r) P_{j}(r)$ when Breit retardation is included in the self-consistent field process. These potential terms can be obtained very efficiently numerically by solving a second-order differential equation (Poisson equation), as a set of two first-order differential equations, with the predictor-corrector method prensented in Sec. 10. 


\section{Numerical solution of the inhomogeneous Dirac-Fock radial equa- tions}

In order to increase the numerical stability, the direct numerical computation of (9.5) is done by shooting techniques. First one chooses a change of variables to make the method more efficient because bound orbitals exhibit a rapid variation near the origin and exponential decay at large distances. One can choose either

$$
t=r_{0} \log (r) \quad \text { or } \quad t=r_{0} \log (r)+b r
$$

The first choice leads to a pure exponential grid, while the second leads to an exponential grid at short distances and to a linear grid at infinity, and is better suited to represent, e.g., Rydberg states. One then takes a linear grid in the new variable $t, t_{n}=n h$ with $h$ ranging from 0.02 to 0.05 . In order to provide the few values needed to start the numerical integration at $r=0$, and to have accurate integrals (for evaluation of the norm for exemple) the wave function is represented by its series expansion at the origin, which is of the form

$$
\left\{\begin{array}{l}
P_{\kappa}(r)=r^{\lambda}\left(p_{0}+p_{1} r+\ldots\right), \\
Q_{\kappa}(r)=r^{\lambda}\left(q_{0}+q_{1} r+\ldots\right),
\end{array}\right.
$$

where $\lambda=\sqrt{\kappa^{2}-(Z \alpha)^{2}}$ if $V_{N}(r)=-Z / r$ is a pure Coulomb potential and $\lambda=|\kappa|$ if $V_{N}(r)$ represents the potential of a finite charge distribution. In this case if $\kappa>0, p_{0}=p_{2}=\ldots=0$ and $q_{1}=q_{3}=\ldots=0$, and if $\kappa<0$, $p_{1}=p_{3}=\ldots=0, q_{0}=q_{2}=\ldots=0$.

Predictor-Corrector Methods.

In the case of the atomic problem, the use of fancy techniques like adaptative grids is not recommended, as it is much more efficient to tabulate all wave functions over the same grid, particularly if other properties like transition probabilities are calculated as well. One then uses well proven differential equation solving techniques like predictor-corrector methods and finite difference schemes. The expansion (10.2) is substituted into the differential equation (9.5) to obtain the coefficients $p_{i}$ and $q_{i}$, for $i>0$. These coefficients are used to generate values for the wave function at the few first $n$ points of the grid, with an arbitrary value of $p_{0}$. Then the value of the function at the next grid point is obtained using the differential equation solver. At infinity the same procedure is used. An exponential approximation of the wave function is made, and the same differential equation solver is used downward to some matching point $r_{m}$, usually chosen close to the classical turning point in the potential $V_{A}(r)$. In the predictor-corrector technique, an approximate value of 
the function at the mesh point $n+1$ is predicted from the known values at the preceding $n$ points. This estimate is inserted in the differential equation to obtain the derivative that in turn is used to correct the first estimate, then the final value may be taken as a linear combination of the predicted and corrected values to increase the accuracy. As an example we consider the five points Adams' method that has been widely selected because of its stability properties [43]. The predicted, corrected and final values are given respectively by:

$$
\begin{aligned}
& p_{n+1}=y_{n}+\left(1901 y_{n}^{\prime}-2774 y_{n-1}^{\prime}+2616 y_{n-2}^{\prime}-1274 y_{n-3}^{\prime}+251 y_{n-4}^{\prime}\right) / 720 \\
& c_{n+1}=y_{n}+\left(251 p_{n+1}^{\prime}+646 y_{n}^{\prime}-264 y_{n-1}^{\prime}+106 y_{n-2}^{\prime}-19 y_{n-3}^{\prime}\right) / 720,(10.3) \\
& y_{n+1}=\left(475 c_{n+}+27 p_{n+1}\right) / 502
\end{aligned}
$$

where $p^{\prime}$ and $y^{\prime}$ stand for the derivatives with respect to the tabulation variable. The linear combination for the final value is defined as to cancel the term of order $h^{6}, h$ being the constant interval step of the mesh. In the above equations, $y$ represents either the large or small component of the radial wave function.

Since one starts with a somewhat arbitrary energy and slope at the origin, the components of the wave function obtained by the preceding method are not continuous. A strategy must be devised to obtain the real eigenenergy and slope at the origin from the numerical solution. In the case of an homogeneous equation, one can simply make the large component continuous by multiplying the wave function by the ratio of the inward and outward values of the large component at the matching point and then change the energy until the small component is continuous, using the default in the norm. To first order the correction to the eigenvalue is

$$
\delta \epsilon=\frac{c P\left(r_{m}\right)\left[Q\left(r_{m}^{-}\right)-Q\left(r_{m}^{+}\right)\right]}{\int_{0}^{\infty}\left[P^{2}(u)+Q^{2}(u)\right] d u},
$$

where $Q\left(r_{m}^{ \pm}\right)$are the solutions from each side of the matching point. One then checks that the solution is the desired one by verifying that it has the right number of nodes.

In the inhomogeneous case such a strategy cannot work. In order to obtain a solution which is continuous everywhere, it is possible to proceed in the following way. One uses the well known fact that the solution of an inhomogeneous differential equation can be written as the sum of a particular solution of the inhomogeneous equation and of the solution of the associated homogeneous equation (in the present case the equation obtained by neglecting the exchange potentials). Thus if $P^{o}$ and $P^{i}$ are respectively the outward and inward solutions for the large component, one obtains, with the same labels for the small 
component:

$$
\left[P_{I}^{o}+a P_{H}^{o}\right]_{r=r_{m}^{-}}=\left[P_{I}^{i}+b P_{H}^{i}\right]_{r=r_{m}^{+}},\left[Q_{I}^{o}+a Q_{H}^{o}\right]_{r=r_{m}^{-}}=\left[Q_{I}^{i}+b Q_{H}^{i}\right]_{r=r_{m}^{+}}
$$

where the subscripts $I$ and $H$ stand for the inhomogeneous and homogeneous solutions. The coefficients $a$ and $b$ can be obtained from the differential equation. Obviously this continuous solution will not be normalized for an arbitrary value of the diagonal parameter $\epsilon_{A, A}$ of Eq.(9.5). The default in the norm is then used to modify $\epsilon_{A, A}$ until the proper eigenvalue is found. This method is very accurate but not very efficient since it requires to solve both the inhomogeneous and the homogeneous equations to obtain a continuous solution.

\section{Finite Differences Methods}

As seen above, the predictor-corrector method has some disadvantages. In the non-relativistic case the Numerov method associated with tail correction [20] provides directly a continuous approximation (the derivative remains discontinuous until the eigenvalue is found). We consider now alternative methods that easily allow to enforce the continuity of one of the two radial components. Let us define the solution at point $n+1$ as:

$$
y_{n+1}=y_{n}+h\left(y_{n}^{\prime}+y_{n+1}^{\prime}\right)+\Delta_{n}
$$

where $\Delta_{n}$ is a difference correction given, in terms of central differences, by:

$$
\Delta_{n}=\frac{-1}{12} \delta^{3} y_{n+\frac{1}{2}}+\frac{1}{120} \delta^{5} y_{n+\frac{1}{2}}
$$

with:

$$
\begin{aligned}
& \delta^{3} y_{n+\frac{1}{2}}=y_{n+2}-3 y_{n+1}+3 y_{n}-y_{n-1} \\
& \delta^{5} y_{n+\frac{1}{2}}=y_{n+3}-5 y_{n+2}+10 y_{n+1}-10 y_{n}+5 y_{n-1}-y_{n-2} .
\end{aligned}
$$

Accurate solutions are required only when self-consistency is reached. Consequently, the difference correction $\Delta_{n}$ can be obtained at each iteration from the wave functions of the previous iteration as it is done for the potential terms. One can then design computationally efficient schemes [7]. We define

$$
\begin{aligned}
a_{n} & =1+\frac{\kappa h}{2} \frac{r_{n}^{\prime}}{r_{n}}, & u_{n} & =\Delta_{n}^{P}+\frac{h}{2}\left[r_{n}^{\prime} X_{n}^{Q}+r_{n+1}^{\prime} X_{n+1}^{Q}\right], \\
b_{n} & =-1+\frac{\kappa h}{2} \frac{r_{n}^{\prime}}{r_{n}}, & v_{n} & =\Delta_{n}^{P}+\frac{h}{2}\left[r_{n}^{\prime} X_{n}^{P}+r_{n+1}^{\prime} X_{n+1}^{P}\right], \\
\varphi_{n} & =\alpha \frac{h}{2}\left[\epsilon_{n}-V_{n}\right] r_{n}^{\prime}, & \theta_{n} & =\frac{h}{\alpha} r_{n}^{\prime}+\theta_{n},
\end{aligned}
$$

where $r^{\prime}$ stands for $d r / d t$ (to take into account the fact that the tabulation variable $t$ is a function of $r$ ) and $X^{P(Q)}=X_{P_{A}\left(Q_{A}\right)}+\sum_{B \neq A} \epsilon_{A, B} P_{B}\left(Q_{B}\right)$. All 
the functions of $r$ are evaluated using wave functions obtained at the previous iteration. Then the system of algebraic equations:

$$
\begin{gathered}
a_{n+1} P_{n+1}-\theta_{n+1} Q_{n+1}+b_{n} P_{n}-\theta_{n} Q_{n}=u_{n}, \\
\varphi_{n+1} P_{n+1}-b_{n+1} Q_{n+1}+\varphi_{n} P_{n}-a_{n} Q_{n}=v_{n}
\end{gathered}
$$

determines $P_{n+1}$ and $Q_{n+1}$ if $P_{n}$ and $Q_{n}$ are known. For the outward integration, this system is solved step by step from near the origin to the matching point after getting the solution at the first point by series expansion. For the inward integration, an elimination process is used by expressing the solution in the matrix form $[M](P Q)=(u v)$ with the matrix $M$ given by:

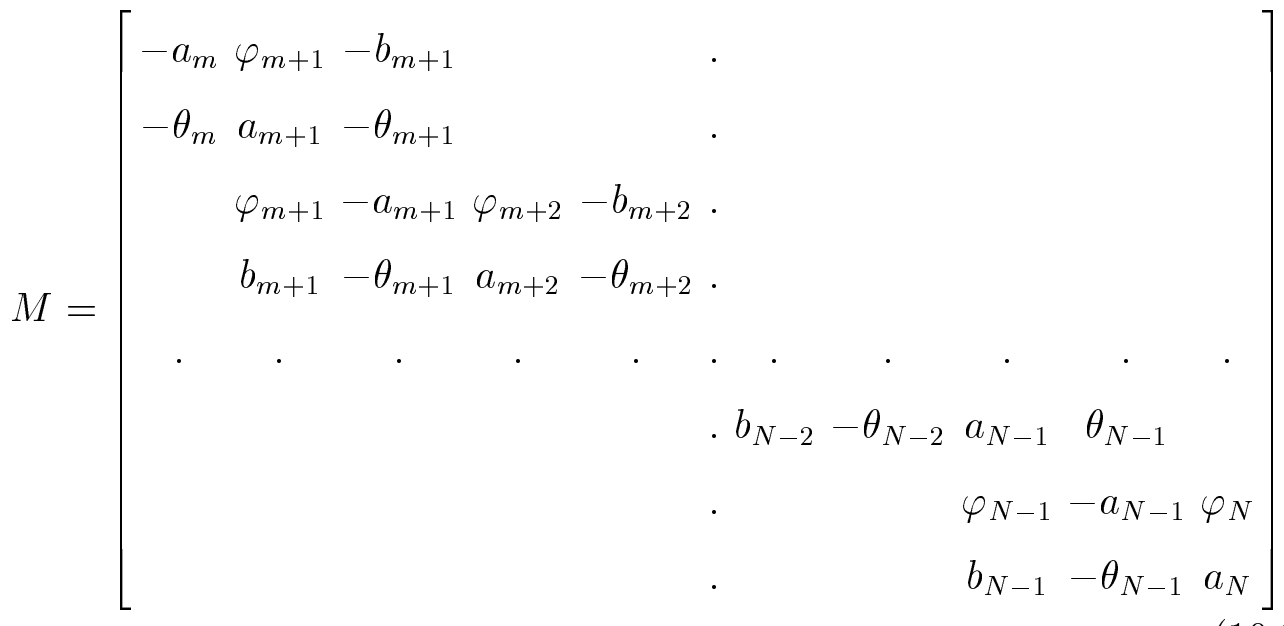

and the two column vectors $(P Q)$ and (uv) defined as:

$$
(P Q)=\left[\begin{array}{c}
Q_{m} \\
P_{m+1} \\
Q_{m+1} \\
P_{m+2} \\
\cdot \\
P_{N-1} \\
Q_{N-1} \\
P_{N}
\end{array}\right] \quad(u v)=\left[\begin{array}{c}
v_{m}-\varphi_{m} P_{m} \\
u_{m}-b_{m} P_{m} \\
v_{m+1} \\
u_{m+1} \\
\\
u_{N-2} \\
v_{N-1}+b_{N} Q_{N} \\
u_{N-1}+\theta_{N} Q_{N}
\end{array}\right] .
$$

As displayed in Eq. (10.11) each row of the matrix $M$ has at most four non-zero elements. To solve this system of equations the matrix $M$ is decomposed into the product of two triangular matrices $M=L T$ in which $L$ is a lower matrix with only three non-zero elements on each row and $T$ an upper matrix with the 
same property. Introducing an intermediate vector $(p q)$ it is possible to solve $L(p q)=(u v)$ for $m, m+1, \ldots, N$ and then $T(P Q)=(p q)$ for $N, N-1, \ldots$ $m$. The last point of tabulation $N$ is determined by the requirement that $P_{N}$ should be lower than a specified small value when assuming $Q_{N}=0$. Thus the number of tabulation points of each orbital is determined automatically during the self-consistency process. This elimination process produces, as written here, a large component $P$ that is continuous everywhere. The discontinuity of the small component at the matching point $r_{m}$ can then be used to adjust the eigenvalue $\epsilon_{A, A}$. In practice this method works very well for occupied orbitals (i.e. orbitals with effective occupations at the Dirac-Fock level $q_{o}^{(A)} \equiv n, n$ integer larger or equal to 1). Yet it is not sufficiently accurate for correlation orbitals and leads to convergence instability. A good strategy [6] is thus to use the accurate predictor-corrector method for the outward integration and the finite differences method with the tail correction for the inward integration. However the accuracy of the inward integration is increased by computing directly the difference correction (10.7) from the wave function being computed rather than from the one from the previous iteration.

\section{Diagonal Lagrange multipliers}

One can use differential techniques, when the obtention of the eigenenergy $\epsilon_{A A}$ is difficult. Their evaluation proceeds as follows. One can obtain the first order variation of the large component $P$ with respect to a change $\Delta \epsilon_{A A}$ of one of the off-diagonal Lagrange multipliers by substituting the development

$$
P\left(\epsilon_{A A}^{0}+\Delta \epsilon_{A A}\right)=P\left(\epsilon_{A A}^{0}\right)+\left.\Delta \epsilon_{A A} \frac{\partial P}{\partial \epsilon_{A A}}\right|_{\epsilon_{A A}=\epsilon_{A A}^{0}}
$$

(and the equivalent one for the small component $Q$ ) into the differential equation (9.5). Defining

$$
p_{A A}=\frac{\partial P}{\partial \epsilon_{A A}}, \quad q_{A A}=\frac{\partial Q}{\partial \epsilon_{A A}}
$$

leads to the new set of differential equations

$$
\left(\begin{array}{cc}
\frac{d}{d r}+\frac{\kappa_{A}}{r} & -\frac{2}{\alpha}+\alpha V_{A}(r) \\
-\alpha V_{A}(r) & \frac{d}{d r}-\frac{\kappa_{A}}{r}
\end{array}\right)\left(\begin{array}{c}
p_{A A}(r) \\
q_{A A}(r)
\end{array}\right)=\alpha \epsilon_{A, A}\left(\begin{array}{c}
q_{A A}(r) \\
-p_{A A}(r)
\end{array}\right)+\alpha\left(\begin{array}{c}
Q_{B}(r) \\
-P_{B}(r)
\end{array}\right)
$$

which is very similar to (9.5), with the replacement of $X_{P_{A}}(r)$ (resp. $X_{Q_{A}}$ ) by $P_{B}(r)$ (resp. $Q_{B}(r)$ ). This system can be solved in $p_{A A}(r)$ and $q_{A A}(r)$ by the above techniques. With this solutions $\Delta \epsilon_{A A}$ can be calculated in first order 
from

$$
\Delta \epsilon_{A A}=\frac{1-\int_{0}^{\infty}\left[P_{A}(r) P_{B}(r)+Q_{A}(r) Q_{B}(r)\right] d r}{2 \int_{0}^{\infty}\left[p_{A A}(r) P_{B}(r)+q_{A A}(r) Q_{B}(r)\right] d r} .
$$

Note that such relations could be established to provide the change in the non-diagonal Lagrange multipliers $\epsilon_{A B}$ as well, if one were to solve for several orbitals of identical symmetry simultaneously.

\section{Off-diagonal Lagrange multipliers}

The self-consistent process outlined in Section 3 requires the evaluation of the off-diagonal Lagrange parameters to satisfy the orthonormality constraint (9.4). As in the non-relativistic case, the off-diagonal Lagrange multiplier between closed ${ }^{1}$ shells can be set to zero, which only amounts to perform a unitary transformation in the subspace of the closed shells. If the generalized occupation numbers $o_{A}$ and $o_{B}$ of two orbitals are different, one can use the symmetry relation

$$
\epsilon_{A B} O_{A}=\epsilon_{B A} O_{B}
$$

and (9.5) to obtain

$$
\begin{aligned}
& \frac{\epsilon_{A B}\left(o_{B}-o_{A}\right)}{o_{B}}=\int_{0}^{\infty}\left[V_{A}(r)-\right.\left.V_{B}(r)\right]\left[P_{A}(r) P_{B}(r)+Q_{A}(r) Q_{B}(r)\right] d r \\
&-\frac{1}{\alpha} \int_{0}^{\infty}\left[X_{Q_{A}}(r) Q_{B}(r)-X_{Q_{B}}(r) Q_{A}(r)\right. \\
&\left.\quad+X_{P_{A}}(r) P_{B}(r)-X_{P_{B}}(r) P_{A}(r)\right] d r
\end{aligned}
$$

This equation shows that many terms will cancel out in the determination of the Lagrange multipliers [e.g., the closed shell contribution to $V_{A}(r)$ and $\left.V_{B}(r)\right]$ and thus provides an accurate method to calculate them provided one retains only the non-zero contributions. If $\left(o_{B}-o_{A}\right) \ll 1$, however, one must use Eqs. (10.15) and (10.16) to evaluate the Lagrange multipliers.

\section{Solution of the inhomogeneous Dirac-Fock equation over a basis set}

It has been found however [30,31] that even the enhanced numerical techniques presented in Sec. 10 would not work for correlation orbitals with very

1 Closed shells are the shell filled with the maximum number of electrons as allowed by the Pauli principle, i.e. $2|\kappa|$. 
small effective occupation, particularly when the contribution of the Breit interaction is used in (9.5). This leads to point out that in the numerical MCDF calculations, the projection operators which should be used according to (1.5) are absent, as they have no explicit expression. A new method has been proposed that retains the advantages of the numerical MCDF. The idea is to expand $P_{A}, Q_{A}, X_{P_{A}}$ and $X_{Q_{A}}$ over a finite basis set, e.g., the one based on the B-spline calculated following the method of Sec. 6, using the full MCDF direct potential $V_{A}(r)$. Let us thus assume that one has a complete set of solutions $\left\{\phi_{1}^{(A)}, \ldots, \phi_{2 n}^{(A)}\right\}$, with eigenvalues $\left\{\epsilon_{1}^{(A)}, \ldots, \epsilon_{2 n}^{(A)}\right\}$ of the homogeneous equation associated to (9.5). One then writes

$$
\left(\begin{array}{c}
P_{A}(r) \\
Q_{A}(r)
\end{array}\right)=\sum_{i=1}^{2 n} s_{i}^{(A)} \phi_{i}^{(A)}(r) \quad \text { and } \quad\left(\begin{array}{c}
X_{P_{A}}(r) \\
X_{Q_{A}}(r)
\end{array}\right)=\sum_{i=1}^{2 n} x_{i}^{(A)} \phi_{i}^{(A)}(r) .
$$

Substituting back into (9.5) and using the orthonormality of the basis set functions, one easily obtains

$$
s_{i}^{(A)}=\frac{x_{i}^{(A)}+\sum_{B \neq A} \epsilon_{A B} s_{i}^{(B)}}{\alpha\left(\epsilon_{1}^{(A)}-\epsilon_{A A}\right)} .
$$

The square of the norm of the solution of (9.5) is then easily obtained as

$$
N\left(\epsilon_{A A}\right)=\sum_{i=1}^{2 n}\left(s_{i}^{(A)}\right)^{2}=\sum_{i=1}^{2 n}\left(\frac{x_{i}^{(A)}+\sum_{B \neq A} \epsilon_{A B} s_{i}^{(B)}}{\alpha\left(\epsilon_{1}^{(A)}-\epsilon_{A A}\right)}\right)^{2} .
$$

One then can calculate the normalized solution of (9.5) if the off-diagonal Lagrange parameters are known, by solving $N\left(\epsilon_{A A}\right)=1$ for $\epsilon_{A A}$. One can notice the interesting feature of (11.3) that the norm of the solution of the inhomogeneous equation (9.5) has a pole for each eigenenergy of the homogeneous equation. This method has the advantage over purely numerical techniques that by restricting the sums in (11.1) to positive energy eigenstates, one can explicitly implement projection operators, thus solving readily the "no-pair" Hamiltonian (1.2), rather than an ill-defined equation. More details on this method and on the evaluation of the off-diagonal Lagrange multipliers can be found in [31].

\section{Chapter 4. Numerical relativistic methods for molecules}

Most of molecular methods that include relativistic corrections are based on the expansion of the molecular orbitals in terms of basis sets (most of the time taken to be Gaussian functions). We shall not review these methods here but refer the interested reader to a book to be published soon [49]. Let us just point 
out that the sometimes observed lack of convergence to upper bounds in the total energy (the so-called variational collapse) is not unambigously related to the Dirac negative energy continuum. Indeed this attractive explanation is unfortunately unable to explain the appearance of spurious solutions. Both the existence of spurious solutions and the lack of convergence to expected levels can be traced back to originate from poor basis sets and bad finite matrix representations of the operators (in particular the kinetic energy). For an extensive discussion see [15]. Numerical methods succesfully used are briefly sketched in the next two paragraphs.

\section{Fully numerical two-dimensional method}

For diatomic molecules, the one-electron Dirac wave functions may be written as

$$
\Phi=\left(\begin{array}{cc}
e^{i(m-1 / 2) \varphi} & \phi_{1}^{L}(\xi, \eta) \\
e^{i(m+1 / 2) \varphi} & \phi_{2}^{L}(\xi, \eta) \\
i e^{i(m-1 / 2) \varphi} & \phi_{3}^{S}(\xi, \eta) \\
i e^{i(m+1 / 2) \varphi} & \phi_{4}^{S}(\xi, \eta)
\end{array}\right)
$$

where $L(S)$ stands for the large (small) component and elliptical coordinates $(\xi, \eta, \varphi)$ are used with:

$$
\xi=\left(r_{1}+r_{2}\right) / R, \quad \eta=\left(r_{1}-r_{2}\right) / R
$$

where $r_{1}$ and $r_{2}$ are the distances between the electron and each of the nucleus, $R$ is the inter-nuclear distance. The third variable $\varphi$ is the azimutal angle around the axis through the nuclei.

As usual for molecular calculations, the variational collapse is avoided by defining the small component in terms of the large one [35]. Starting from the Dirac equation in a local potential $V$ one possibility is to use:

$$
\phi^{S}=c \boldsymbol{\sigma} \cdot \boldsymbol{p} \phi^{L} /\left[2 c^{2}+E-V\right] .
$$

After this substitution, the large component is given as solution of a second order differential equation that can be solved using well known relaxation methods [55].

For efficiency, the distribution of integration points must be chosen as to accumulate points where the functions are rapidly varying. It was found that 
the transformation,

$$
\mu=\operatorname{arccosh}(\xi), \quad \nu=\operatorname{arccosh}(\eta)
$$

which yields a quadratic distribution of points near the nuclei, is some kind of optimum to reduce the number of points needed to achieve a given accuracy. Then the derivatives of the Laplace operator are approximated by $n$-point finite differences. In so doing, the differential equations are replaced by a set of linear equations that can be written in a matrix form as

$$
(\boldsymbol{A}-E \boldsymbol{S}) X=B
$$

where the matrix A, that represents the direct part of the Fock operator, is diagonal dominant but has non-diagonal elements arising from the discretization of the Laplace operator. Here E is the energy eigenvalue, $\mathrm{S}$ is the overlap diagonal matrix and $\mathrm{B}$ a vector due to the exchange part of the Fock operator whose values change during iterations. Then the relaxation method can be viewed as an iterative method to find the $x_{i}$ component of $X$ such that

$$
(\boldsymbol{A}-E \boldsymbol{S}) x_{i}=b_{i}
$$

each iteration $n$ being associated with a linear combination of the initial and final estimate of $x_{i}$ at iteration $n-1$, i.e.

$$
x_{i}^{\text {initial }_{n+1}}=(1-\omega) x_{i}^{\text {initial }_{n}}+\omega x_{i}^{\text {final }_{n}} .
$$

It was found that with overrelaxation (i.e. $\omega>1$ ), the method may be slow in convergence but it is quite stable. Applications of the method outlined above may be found in [53] and in references therein.

\section{Numerical integrations with linear combinations of atomic or- bitals}

A widely used approximation in molecular calculations is to expand the molecular orbitals as a linear combination of atomic orbitals. If these atomic orbitals are chosen as the numerical solutions of some kind of Dirac-Fock atomic calculations, then small basis sets are sufficient to achieve good accuracy. The main disavantage of this choice is that all multi-dimensional integrals have to be calculated numerically. This is compensated by two advantages: first the kinetic energy contribution can be computed by a single integral using the atomic Dirac equations (thus avoiding numerical differenciation), second, by including only positive energy atomic wave functions, no "variational collapse" will occur. 
In this method, the molecular wave functions $\psi$ are expanded in terms of symmetry molecular orbitals $\chi$ as:

$$
\psi_{\lambda}=\sum_{\nu} c_{\nu}^{\lambda} \chi^{\nu}
$$

while the symmetry molecular orbitals $\chi$ are taken to be linear combinations of atomic orbitals $\varphi$ :

$$
\chi^{\nu}=\sum_{i} d_{i}^{\nu} \varphi^{i}
$$

The coefficients $d_{i}^{\nu}$ are given by the symmetry of the molecular orbital and are obtained from the irreducible representations of the double point groups. Computing all necessary integrals (overlaps, matrix elements of the Dirac operator, the Coulomb interaction, etc...) the Dirac-Fock equations are reduced to a generalized matrix eigenvalue problem that determines both the eigenvalues and the $c_{\nu}^{\lambda}$ coefficients of equation (13.1).

To compute the various matrix elements in the case of diatomic molecules, Sepp et al. [50] used Gauss-Laguerre and Gauss-Legendre integration schemes on a grid of points defined by the same variables as those of Eq. (12.4). Unfortunately this approach is not easy to extend beyond diatomic molecules and other methods have to be implemented. It has beeen shown, see for example [44], that the adaptation to molecules of the so-called Discrete Variational Method (DVM) developped for solid state calculations [17] may be both efficient and accurate. The DVM may be viewed as performing a multidimensional integral via a weighted sum of sampling points, i.e. to compute a matrix element $<f>$ by:

$$
<f>=\sum_{n=1}^{N} \omega\left(r_{i}\right) f\left(r_{i}\right)
$$

where the weight function $\omega\left(r_{i}\right)$ can be considered as an integration weight corresponding to a local volume per point. This function is also constrained to force the error momenta to vanish on the grid points following the work of Haselgrove [27]. Furthermore the set of the sampling points $\left[r_{i}\right]$ must be chosen to preserve the symmetries of the system under configuration (this is accomplished by taking a set of sampling points that includes all points $R r_{i}$, $R$ standing for operations of the symmetry group). A full description of the DVM can be found in the references given above. 


\section{References}

[1] W.E. BAyliss and S. J. PeEL. Stable variational calculations with the Dirac Hamiltonian. Phys. Rev. A, 28 (4) (1983), p. 2552-2554.

[2] T. BEIER. The $g_{j}$ Factor of a Bound Electron and the Hyperfine Structure Splitting in Hydrogenlike Ions. Physics Reports 339(2-3) (2000), p. 79-213.

[3] G. E. Brown and D. E. Ravenhall. On the Interaction of Two Electrons. Proc. R. Soc. London, Ser A 208(1951), p. 552-559.

[4] Ch. Chang, J.-P. Pelissier and Ph. Durand. Regular two-Component Paulilike Effective Hamiltonians in Dirac Theory. Phys. Scripta 34 (1986), p. 394-404.

[5] S.N. Datta and G. Deviah. The minimax technique in relativistic HartreeFock calculations. Pramana 30(5) (1988), p. 393-416.

[6] J. P. Desclaux. A Relativistic Multiconfiguration Dirac-Fock Package. In Methods and Techniques in Computational Chemistry Clementi. E. Ed A: Small Systems STEF, 1993.

[7] J. P. Desclaux, D. F. Mayers and F. O’Brien. Relativistic Atomic Wavefunction. J. Phys. B: At. Mol. Opt. Phys. 4(1971), p. 631-642.

[8] J. Dolbeault, M.J. Esteban and E. SÉrÉ. Variational characterization for eigenvalues of Dirac operators. Calc. Var. and P.D.E. 10 (2000), p. 321-347.

[9] J. Dolbeault, M.J. Esteban and E. SÉRÉ. On the eigenvalues of operators with gaps. Application to Dirac operators. J. Funct. Anal. 174 (2000), p. 208226.

[10] J. Dolbeault, M.J. Esteban, E. SÉré and M. Vanbreugel. Minimization methods for the one-particle Dirac equation. Phys. Rev. Letters 85(19) (2000), p. $4020-4023$

[11] G.W.F. Drake and S.P. Goldman. Application of discrete-basis-set methods to the Dirac equation. Phys. Rev. A 23 (1981), p. 2093-2098.

[12] G.W.F. Drake and S.P. Goldman. Relativistic Sturmian and finite basis set methods in atomic physics. Adv. Atomic Molecular Phys. 23 (1988), p. 23-29.

[13] Ph. Durand. Transformation du Hamiltonien de Dirac en Hamiltoniens variationnels de type Pauli. Application à des atomes hydrogenoïdes. C. R. Acad. Sc. Paris 303 , série II, numéro 2 (1986), p. 119-124.

[14] Ph. DurAnd and J.-P. MALRIEU. Effective Hamiltonians and pseudo-potentials as tools for rigorous modelling. In Ab initio methods in Quantum Chemistry I. K.P. Lawley ed.. J. Wiley \& Sons, 1987.

[15] K.G. Dyall, I.P. Grant and S. Wilson. Matrix representation of operator products. J. Phys. B: At. Mol. Phys. 17 (1984), p. 493-503 
[16] M. I. Eides, H. Grotch and V. A. Shelyuto. Theory of Light Hydrogenlike Atoms. Phy. Rep. 342(2-3) (2001), p. 63-261.

[17] D. E. Ellis and G. S. Painter. Discrete Variational Method for the EnergyBand Problem with General Crystal Potentials. Phys. Rev. B 2(8) (1970), p. 2887-2898.

[18] M.J. Esteban and E. SÉRÉ. Existence and multiplicity of solutions for linear and nonlinear Dirac problems. Partial Differential Equations and Their Applications. CRM Proceedings and Lecture Notes, vol. 12. Eds. P.C. Greiner, V. Ivrii, L.A. Seco and C. Sulem. AMS, 1997.

[19] L.L. Foldy and S.A. Wouthuysen. On the Dirac theory of spin-1/2 particles and its nonrelativistic limit. Phys. Rev. 78 (1950), p. 29-36.

[20] C. Froese Fischer. The Hartree-Fock Method for Atoms. Wiley, 1977.

[21] I.P. Grant. Conditions for convergence of variational solutions of Dirac's equation in a finite basis. Phys. Rev. A 25(2) (1982), p. 1230-1232.

[22] I.P. Grant. Notes on Basis Sets for Relativistic Atomic Structure and QED. A.I.P. Conf. Proc. 189 (1989), p. 235-253. Ed. P.J. Mohr, W.R. Johnson, J. Sucher.

[23] I. P. Grant. Relativistic Atomic Structure Calculations. Meth. Comp. Chem. 2 (1987), p. 132.

[24] I. P. Grant and H. M. Quiney. Foundation of the Relativistic Theory of Atomic and Molecular Structure. Adv. At. Mol. Phys. 23(1988), p. 37-86.

[25] M. Griesemer, R.T. Lewis and H. Siedentop. A minimax principle in spectral gaps: Dirac operators with Coulomb potentials. Doc. Math. 4 (1999), 275-283 (electronic).

[26] M. Griesemer and H. Siedentop. A minimax principle for the eigenvalues in spectral gaps. J. London Math. Soc. (2) 60 no. 2 (1999), p. 490-500.

[27] C. B. Haselgrove. A method for numerical integration. Math. Comput. 15 (1961), p. 323-337.

[28] J. L. Heully, I. Lindgren, E. Lindroth, S. Lundqvist and A. M. MÅrTensson-Pendrill. Diagonalisation of the Dirac Hamiltonian as a Basis for a Relativistic Many-Body Procedure. J. Phys. B: At. Mol. Phys. 19(1986), p. $2799-2815$.

[29] R.N. Hill and C. Krauthauser. A solution to the problem of variational collapse for the one-particle Dirac equation. Phys. Rev. Lett. 72(14) (1994), p. 2151-2154.

[30] P. Indelicato and J. P. Desclaux. Projection Operators in the Multiconfiguration Dirac-Fock Method. Phys. Scr. T46(1993), p. 110-114. 
[31] P. Indelicato. Projection Operators in Multiconfiguration Dirac-Fock Calculations. Application to the Ground State of Heliumlike Ions. Phys. Rev. A 51(2) (1995), p. 1132-1145.

[32] W. R. Johnson, S. Blundell and J. Sapirstein. Finite Basis Sets for Dirac Equation Constructed from B splines. Phys. Rev. A 37(2) (1988), p. 307-315.

[33] W. R. Johnson and C. D. Lin. Relativistic Random Phase Approximation Applied to Atoms of the He Isoelectronic Sequence. Phys. Rev. A 14(2) (1976), p. $565-575$.

[34] J. B. Kogut. Lattice Gauge Theory Approach to Quantum Chromodynamics. Rev. Mod. Phys. 55(3) (1983), p. 775-836.

[35] W. Kutzelnigg. Basis Set Expansion of the Dirac Operator without Variational Collapse. Int. J. Quant. Chem. 25 (1984), p. 107-129.

[36] W. Kutzelnigg. Perturbation theory of relativistic corrections 2. Analysis and classification of known and other possible methods. Z. Phys. D - Atoms, Molecules and Clusters 15 (1990), p. 27-50.

[37] W. Kutzelnigg. Effective Hamiltonians for degenerate and quasidegenerate direct perturbation theory of relativistic effects. J. Chem. Phys. 110(17) (1999), p. 8283-8294.

[38] S.-H. LEE. A new basis set for the radial Dirac equation. Preprint 2001.

[39] E. van Lenthe, R. van Leeuwen, E.J. Baerends and J.G. Snijders. Relativistic regular two-component Hamiltonians. In New challenges in computational Quantum Chemistry. R. Broek et al ed. Publications Dept. Chem. Phys. and Material sciences. University of Groningen, 1994.

[40] R. van Leeuwen, E. van Lenthe, E.J. Baerends and J.G. Snijders. Exact solutions of regular approximate relativistic wave equations for hydrogen-like atoms. J. Chem. Phys. 101(2) (1994), p. 1272-1281.

[41] I. Lindgren and J. Morrison. Atomic Many-Body Theory. Springer, 1982.

[42] P. J. Mohr, G. Plunien and G. Soff. Qed Corrections in Heavy Atoms. Phy. Rep. 293(5\&6) (1998), p. 227-372.

[43] W. H. Press, B. P. Flannery, S. A. Teukolsky and W. T. Vetterling. Numerical Recipes. Cambridge University Press, 1986.

[44] A. Rosen and D. E. Ellis. Relativistic molecular calculations in the DiracSlater model. J. Chem. Phys. 62 (1975), p. 3039-3049.

[45] A. Rutkowski and W.H.E. Schwarz. Effective Hamiltonian for neardegenerate states in direct relativistic perturbation theory. I. Formalism. J. Chem. Phys. 104(21) (1996), p. 8546-8552.

[46] A. Rutkowski. Iterative solution of the one-electron Dirac equation based on the Bloch equation of the 'direct perturbation theory'. Chem. Phys. Letters 307 (1999), p. 259-264. 
[47] S. Salomonson and P. “'Oster. Relativistic All-Order Pair Functions from a Discretized Single-Particle Dirac Hamiltonian. Phys. Rev. A 40(10) (1989), p. 5548-5558.

[48] S. Salomonson and P. Öster. Solution of the Pair Equation Using a Finite Discrete Spectrum. Phys. Rev. A 40(10) (1989), p. 5559-5567.

[49] P. Schwerdtfeger Ed. Relativistic Electronic Structure Theory. Part 1 Fundamental Aspects Elsevier (2002)

[50] W. D. Sepp, D. Kolb, W. Sengler, H. Hartung and B. Fricke. Relativistic Dirac-Fock-Slater program to calculate potential-energy curves for diatomic molecules. Phys. Rev. A 33 (1986), p. 3679-3687.

[51] R. Stacey. Eliminating Lattice Fermion Doubling. Phys. Rev. D 26(2) (1982), p. $468-472$.

[52] J. Sucher. Foundation of the Relativistic Theory of Many-Electron Atoms. Phys. Rev. A 22(2) (1980), p. 348-362.

[53] D. Sundholm, P. Pyykkö and L. LaAksonen. Two-dimensional fully numerical solutions of second-order Dirac equations for diatomic molecules. Part 3 Phys. Scr. 36 (1987), p. 400-402.

[54] J.D. Talman. Minimax principle for the Dirac equation. Phys. Rev. Lett. 57(9) (1986), p. 1091-1094.

[55] R. S. VArga. Matrix iterative analysis. Prentice-Hall, Englewoods Cliffs, N.J., 1963.

[56] H. Wallmeier and W. Kutzelnigg. Use of the squared Dirac operator in variational relativistic calculations. Chem. Phys. Lett. 78(2) (1981), p. 341346.

[57] K. G. Wilson. Confinement of Quarks. Phys. Rev. D 10(8) (1974), p. 24452459.

[58] J. Wood, I.P. Grant and S. Wilson. The Dirac equation in the algebraic approximation: IV. Application of the partitioning technique. J. Phys. B: At. Mol. Phys. 18 (1985), p. 3027-3041. 


\section{Index}

- 2-spinor, 9

- B-splines, 10, 14

- adaptative grids, 21

- angular momentum, 6

- atomic models, 1

- bound states, 3

- Breit interaction, 27

- configuration state functions, 6

- constraints, 1,18

- continuous spectrum, 1

- Coulomb potential, 19, 21

- diagonal Lagrange multipliers, 25

- Dirac equation, 1, 13

- Dirac operator, 7

- Dirac-Fock, 1, 3, 4, 19, 29

- discrete variational method, 30

- effective Hamiltonians, 1, 8, 15, 16

- effective occupation, 20

- eigenvalues, $1,7,10,17$

- essential spectrum, 7, 17

- exchange potentials, 19

- exchange terms, 19

- fine structure constant, 3

- finite basis, 1

- finite difference schemes, 21

- finite differences methods, 23

- Foldy and Wouthuysen, 16

- Galerkin method, 14

- gap, 7

- generalized Bloch equation, 5

- Hartree-Fock, 20

- heavy atoms, 2

- highly charged ions, 2

- inner shells, 2

- kinetic balance, 10

- linear combination of atomic orbitals, 29 
- logarithmic lattice, 12

- MCDF method, 1

- min-max, 1, 17

- minimization, 1, 18

- mixing coefficients, 20

- molecular methods, 27

- molecular models, 1

- Multiconfiguration Dirac-Fock, 4

- Multiconfiguration Dirack-Fock method, 6

- Muticonfiguration Dirac-Fock method, 19, 20

- no-pair Hamiltonian, 1, 2, 27

- numerical basis sets, 1, 11, 14

- occupation numbers, 26

- off-diagonal Lagrange multipliers, 26, 27

- partitioning, 9, 16

- Pauli matrices, 9

- perturbation theory, $1,8,15$

- predictor-corrector methods, 21

- QED, 2, 3

- Rayleigh quotient, 1, 8, 17

- Rayleigh-Schrödinger perturbation theory, 4

- Relativistic Many-Body perturbation theory, 3, 4

- relativistic models, 1

- Relativistic Random Phase Approximation, 1, 4, 5

- Rydberg states, 21

- self-consistent field, 20

- Slater determinants, 6

- Slater type orbitals, 10

- spurious states, 1, 7, 10, 28

- Thomas-Fermi, 20

- variational collapse, 1, 7, 28, 29

- variational methods, $1,8,17$ 